\title{
Multifaceted regulation and functions of YAP/TAZ in tumors (Review)
}

\author{
HUIRONG LIU ${ }^{1 *}$, SUYA DU $^{2^{*}}$, TIANTIAN LEI ${ }^{2^{*}}$, HAILIAN WANG ${ }^{1}$, \\ $\mathrm{XIA} \mathrm{HE}^{3}$, RONGSHENG TONG ${ }^{3}$ and YI WANG ${ }^{3}$
}

\author{
${ }^{1}$ Center of Organ Transplantation, Sichuan Academy of Medical Science and Sichuan Provincial \\ People's Hospital, Chengdu, Sichuan 610072; ${ }^{2}$ School of Medicine, University of Electronic Science \\ and Technology of China Chengdu, Sichuan $610054 ;{ }^{3}$ Personalized Drug Therapy Key Laboratory of \\ Sichuan Province, Department of Pharmacy, Sichuan Academy of Medical Science and \\ Sichuan Provincial People's Hospital, Chengdu, Sichuan 610072, P.R. China
}

Received November 10, 2017; Accepted April 19, 2018

DOI: 10.3892/or.2018.6423

\begin{abstract}
The Hippo pathway, initially identified through screenings for mutant tumor suppressors in Drosophila, is an evolutionarily conserved signaling pathway that controls organ size by regulating cell proliferation and apoptosis. Abnormal regulation of the Hippo pathway may lead to cancer in mammals. As the major downstream effectors of the Hippo pathway, unphosphorylated Yes-associated protein (YAP) and its homolog transcriptional co-activator TAZ (also called WWTR1) (hereafter called YAP/TAZ) are translocated into the nucleus. In the nucleus, in order to induce target gene expression, YAP/TAZ bind to the TEA domain (TEAD) proteins, and this binding subsequently promotes cell proliferation and inhibits apoptosis. In contrast, as key regulators of tumorigenesis and development, YAP/TAZ are phosphorylated and regulated by multiple molecules and pathways including Lats1/2 of Hippo, Wnt and G-protein-coupled receptor (GPCR) signaling, with a regulatory role in cell physiology, tumor cell development and pathological abnormalities simultaneously. In particular, the crucial role of YAP/ TAZ in tumors ensures their potential as targets in designing anticancer drugs. To date, mounting research has elucidated the suppression of YAP/TAZ via effective inhibitors, which significantly highlights their application in cancer treatment. In the present review, we focus on the functions of YAP/TAZ in cancer, discuss their potential as new therapeutic target for
\end{abstract}

Correspondence to: Dr Yi Wang or Dr Rongsheng Tong, Personalized Drug Therapy Key Laboratory of Sichuan Province, Department of Pharmacy, Sichuan Academy of Medical Science and Sichuan Provincial People's Hospital, 32 First Ring Road, Chengdu, Sichuan 610072, P.R. China

E-mail:w_yi@yahoo.com

E-mail: tongrs@126.com

*Contributed equally

Key words: Hippo pathway, YAP/TAZ, carcinogenesis, oncotherapy tumor treatment, and provide valuable suggestions for further study in this field.

\author{
Contents \\ 1. Introduction \\ 2. Hippo pathway in mammals \\ 3. Structural and functional characteristics of YAP/TAZ \\ 4. YAP/TAZ in cancer \\ 5. YAP/TAZ in oncotherapy \\ 6. Conclusion
}

\section{Introduction}

The precise regulation of cell proliferation and apoptosis plays an important role in maintaining an appropriate organ size during organ development and tissue homeostasis during postnatal life (1). The Hippo pathway was initially identified in Drosophila through genetic mosaic screening. As an evolutionarily and functionally conserved signaling network, the Hippo pathway has been discovered to participate in controlling organ size by regulating cell proliferation and apoptosis (2-5).

Following these pioneering discoveries, research concerning the Hippo pathway in mammalian tissues has currently become a burgeoning field $(2,3)$. Generally, in Drosophila, the terminal effector component of the Hippo pathway is a transcription co-activator, named Yorkie (4). However in mammalians, Yes-associated protein (YAP) and its homolog transcriptional co-activator TAZ (also called WWTR1) with PDZ-binding motif are key downstream terminal effectors of the Hippo pathway (5). In normal tissue, YAP/TAZ proteins are phosphorylated at specific serine residues in order to confine their subsequent degradation in the cytoplasm, which consists of the final effect of the Hippo pathway on YAP/TAZ proteins. However in tumors, YAP/TAZ proteins are translocated into the nucleus where they bind to TEA domain (TEAD) proteins. This eventually results in 
cell proliferation, evasion of apoptosis and amplification of progenitor/stem cells for the promotion of organ size (6-8).

Increasing evidence shows that alterations to the Hippo pathway are significantly associated with cancer development $(9,10)$. A high percentage of patients suffering from liver cancer, breast cancer or cancer of the pharynx are reported to harbor causative overexpression of YAP/TAZ genes (11). Moreover, aberrant expression of YAP/TAZ has been demonstrated to be an independent prognostic predictor and indicator of rapid proliferation, metastasis and poor survival outcome of patients with colorectal cancer (CRC) (12).

Herein, we review the functions of YAP/TAZ on tumorigenesis, cell proliferation, metastasis and apoptosis. Furthermore, the importance of YAP/TAZ in cancer treatment is also highlighted in light of the interactive molecular pathways noted among Hippo, Wnt and G-protein-coupled receptor (GPCR) in the regulation of tumor progression and drug resistance. Briefly, we focused on the considerable role of YAP/TAZ in oncotherapy, illuminating their promising application potential as new drug targets for tumor therapeutic intervention.

\section{Hippo pathway in mammals}

The Hippo pathway, conserved in mammals, has received immense research attention in recent years (4). The study from fruit flies to humans shows that the Hippo pathway is highly conserved under normal conditions and functions as a means of inhibiting cancer development (13). Basically, the Hippo pathway consists of three parts: upstream regulatory elements (NF2, Mel), core components (mainly Mst1/2, Lats1/2) and downstream effector molecules (YAP/TAZ). Meanwhile, the central components of the Hippo pathway comprise a regulatory serine-threonine kinase module and a transcriptional module (14) (Table I). In detail, the kinase module includes mammalian STE20-like protein kinase 1 (Mst1, also known as Stk4) and Mst2 (also known as Stk3), as well as large tumor suppressor 1 (Lats1) and Lats2 $(11,15)$. The transcriptional module includes YAP and TAZ. They are two closely related paralogues which primarily mediate the downstream effects of the Hippo pathway via a feedback mechanism. It is now widely acknowledged that the components of the kinase module are tumor suppressors and those of the transcriptional module are oncogenes $(11,16)$.

In normal tissue, Mst1/2, activated by certain upstream signals, can phosphorylate and activate the substrate Lats1/2 (17). Subsequently, the activated Lats1/2 can directly phosphorylate downstream effector molecules YAP/TAZ In addition, the phosphorylated YAP/TAZ interacts with the 14-3-3 protein, resulting in the cytoplasm retention of YAP/ TAZ. Consequently, phosphorylated YAP/TAZ may lose their function as transcription cofactors, which leads to ubiquitinmediated proteasomal degradation $(18,19)$. On the contrary, YAP/TAZ can function as transcriptional co-activators that shuttle between the cytoplasm and nucleus. In the nucleus, they induce expression of cell-proliferative and anti-apoptotic genes via interacting with transcriptional factors, particularly the TEA domain (TEAD) $(6,20,21)$. In short, the Hippo pathway is an important pathway to maintain the homeostasis of cell proliferation and apoptosis (22). Noteworthy, deactivation of
Table I. Elements of the Hippo pathway.

\begin{tabular}{ll}
\hline Location & \multicolumn{1}{c}{ Elements } \\
\hline Upstream & NF2 and Mel \\
Core components & Mainly Mst1/2, Lats1/2 \\
Downstream & YAP/TAZ \\
Central components & Serine-threonine kinase module \\
& (Mst1/2, Lats1/2), a transcriptional \\
& module (YAP/TAZ)
\end{tabular}

the Hippo pathway and the upregulation of YAP/TAZ have been frequently observed in many human cancers $(10,23)$.

Based on the aforementioned analysis, we regard the Hippo kinase module as a switch button. When Hippo kinase module is 'on', LATS1/LATS2 phosphorylates and inactivates YAP/TAZ, thus the output gene production is turned off. Oppositely, when the kinase module is 'off', hypophosphorylated YAP/TAZ are translocated into the nucleus and induce the expression of target genes (Fig. 1).

\section{Structural and functional characteristics of YAP/TAZ}

The YAP1 (Yes-associated protein 1) gene, located on chromosome 11q22 in humans and having a $65-\mathrm{kDa}$ molecular mass (known as YAP or YAP65), is ubiquitously expressed in human tissues throughout the developmental process (24-26). To date, eight splicing isoforms of the YAP1 gene product (YAP1-1 $\alpha, \beta, \gamma, \delta$ and YAP1-2 $\alpha, \beta, \gamma$ and $\delta$ ) have been initially identified in humans and are regarded as YAP1 and YAP2, which differ by the presence of an extra 38 amino acids that encode the WW domain (27). Although the existence of YAP1 (with one WW domain) and YAP2 (with two WW domains) isoforms has been previously unveiled, there are few studies that separate these two types of isoforms in the research of the Hippo pathway (24). As an oncogene, YAP is amplified in various human cancers, which leads to abnormalities of the Hippo-YAP pathway and induces an imbalance between cell proliferation and apoptosis (28). Research in vitro has demonstrated that YAP2 is the predominantly expressed YAP isoform in both ovarian surface epithelium and epithelial ovarian cancers (29). Notably, whether YAP has biological activity or not depends on the location of YAP in cells. Phosphorylated YAP binds to the 14-3-3 protein in the cytoplasm, leading to its cytoplasmic retention and inactivation (30). As for the TAZ gene, it is located on 3q23-q24 and encodes a 43-kDa protein (31) with abundant expression in various tissues except for thymus and peripheral blood leukocytes and amplification in many human cancers (32). However, it is also known as WWTR1 (WW-domain containing transcriptional regulator 1) and is similarly identified as a 14-3-3 binding protein as well.

Structurally, YAP and TAZ share nearly half of the amino acid sequence and have very similar topology features. YAP protein, consisting of 488 amino acids, has a TEAD-binding region (TB), two WW domains (two conserved tryptophan/W residues separated by 20-23 amino acids), an SH3-binding motif, a coiled-coil domain, a transcription activation 


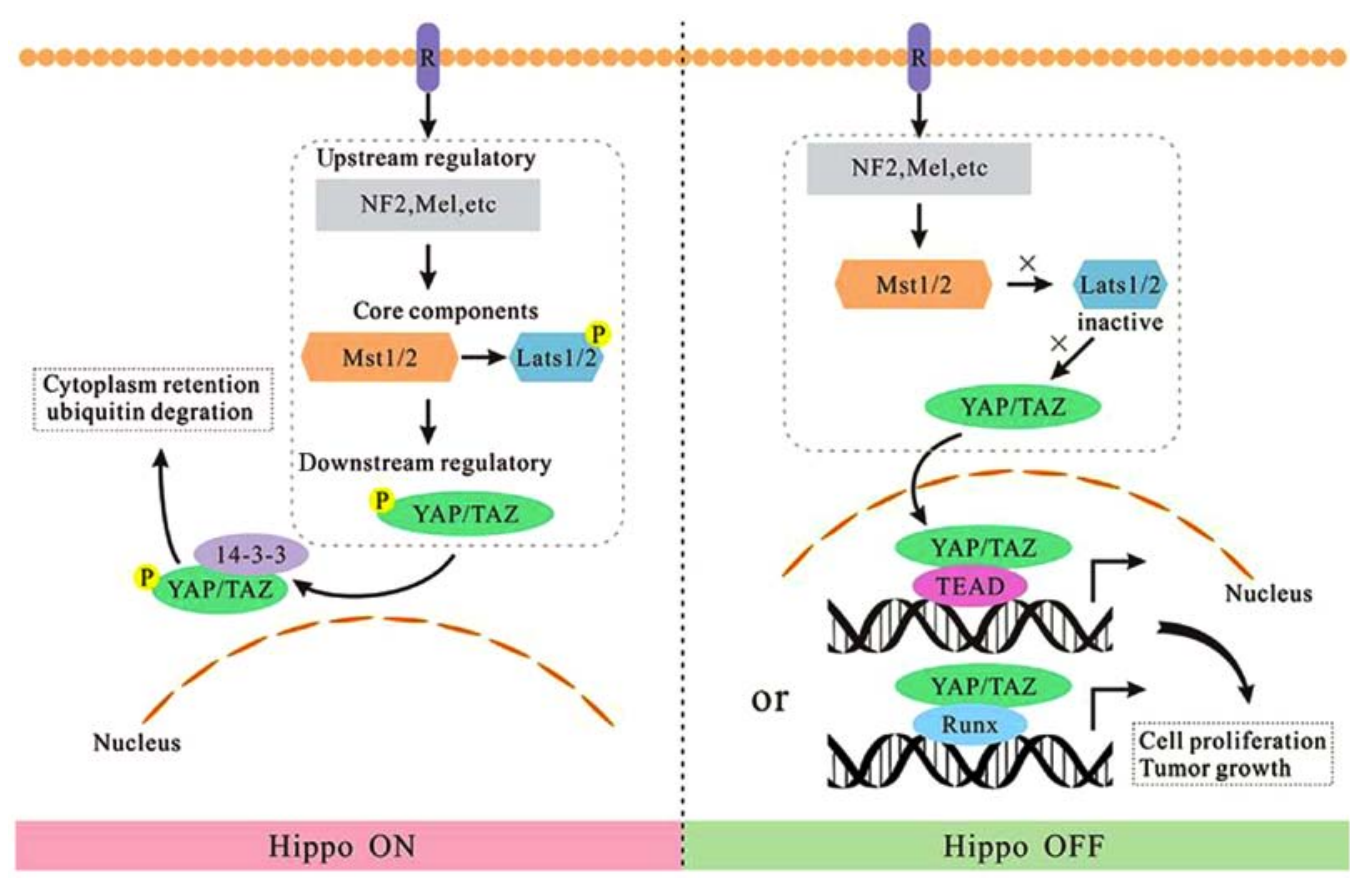

Figure 1. Hippo pathway. 'Hippo On' results in the phosphorylation and inactivation of TAP/TAZ via Lats1/2, ultimately leading to the cytoplasmic retention of TAP/TAZ (left). Oppositely, 'Hippo Off' abrogates the inactivation of TAP/TAZ, thus they are translocated into the nucleus to induce cell proliferation and tumor growth (right). P, phosphorylation; R, receptors; $\downarrow$, activation; $\mathrm{x}$, block.
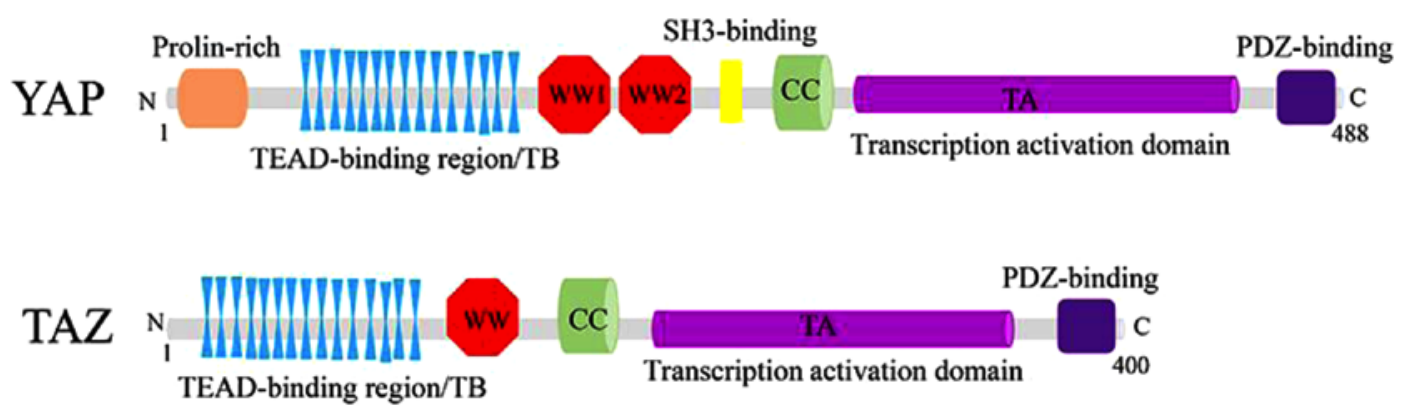

Figure 2. Structures of YAP/TAZ. YAP protein consists of a TEAD-binding region, two WW domains, an SH3-binding motif, a coiled-coil domain, a transcription activation domain, an N-terminal proline-rich domain and a C-terminal PDZ-binding motif (above). Similarly, TAZ protein consists of a TEADbinding region, only one WW domain, a coiled-coil domain, a transcription activation domain and a C-terminal PDZ-binding motif, without SH3-binding motif and proline-rich domain compared with YAP (below). TEAD, YAP/TAZ binds to TEA domain.

domain, an N-terminal proline-rich domain, and a C-terminal PDZ-binding motif. Whereas TAZ protein, consisting of 400 amino acids, has a similar domain organization with YAP, although it lacks the second WW domain, the SH3-binding motif and the proline-rich domain (33,34) (Fig. 2).

Functionally, the WW domains of YAP and TAZ are shown to interact with PPXY motifs of various transcriptional factors (35). The TB domains recognize transcriptional factors, the TEAD family, and activate the expression of target genes. In addition, the 14-3-3 binding motif is also crucial for the regulation of YAP and TAZ (36). In other words, both YAP and TAZ serve as transcriptional co-activators and share various transcriptional factor partners (37). However, there are still various studies indicating that YAP/TAZ have their own unique target transcriptional factors, such as ErbB4 and p73 for YAP and PPAR $\gamma$, Pax3, TBX5 and TTF-1 for TAZ. These different transcriptional factors may contribute to the distinct functions of YAP and TAZ (38-42).

\section{YAP/TAZ in cancer}

YAP/TAZ as biomechanic mediators. It is widely recognized that biomechanics is an important regulator of cell physiology and a pivot in cell development and pathological abnormalities (43). YAP/TAZ, two proto-oncogene proteins, are able to respond to complex physical milieu characterized by the rigidity of extracellular matrix (ECM), mechanical stretching, cell geometry and status of the actin cytoskeleton (44).

It was revealed that breast cancer has elevated tissue stiffness due to the alteration of the ECM (45). Nevertheless, the softening of the tumor microenvironment may contribute to the alleviation of tumor growth and progression. Intriguingly, remodeling of the ECM is partly dependent on YAP activity. In cancer-associated fibroblasts (CAFs), the activation of YAP promotes matrix stiffening through extensive deposition of collagen. Subsequently, the YAP-induced matrix stiffening creates tension within CAFs, leading to the activation of Src 
kinase and the nuclear translocation of YAP. This is conducive to further matrix stiffening (46), thereby establishing a selfenhancing loop during tumorigenesis. Beyond that, mechanical stretching can indeed induce the entry of YAP/TAZ into the nucleus, stimulating the proliferation of contact-inhibited mammary epithelial cells (47). In detail, mechanical stretching is able to override CIP (contact inhibition of proliferation) via YAP/TAZ, although CIP is deregulated in cancer (48). Namely, this is one of the major hallmarks of cell neoplastic transformation.

Crucially, in tumors, the extensive diversity of cell geometry regulates cell proliferation, which is identified by YAP/ TAZ $(49,50)$. However, it is not clear how YAP/TAZ respond to the diversity of cell geometry in cancer. It has been shown that the adhesion sites of YAP/TAZ and their associated F-actin cytoskeleton are affected differently in rounded cells and spread cells $(51,52)$. Moreover, the regulation of YAP/TAZ localization by mechanical stress also depends on F-actin and Rho family GTPases $(53,54)$. Induced actin polymerization by the overexpression of F-actin nucleator precisely correlates with activation of YAP/TAZ (55). Along with these clues, we may explain how YAP/TAZ interact with the F-actin cytoskeleton and sense cell geometry, which eventually leads to the acceleration of tumor cell proliferation. Furthermore, the pro-fibrotic microenvironment of tumors characterized by enhanced stiffness stimulates mesenchymal stromal cells (MSCs) to express $\alpha$-smooth muscle actin ( $\alpha$-SMA). And $\alpha$-SMA incorporates into hMSC stress fibers and promotes downstream translocation of YAP/TAZ transcription factors into the nucleus. Moreover, the nuclear localization of YAP/TAZ is positively correlated with $\alpha$-SMA-expressing stromal cells of adiposarcoma and osteosarcoma (56), further suggesting the significant interaction between YAP/TAZ and biomechanic indicators.

YAP/TAZ as modulators of the Wnt pathway. The canonical Wnt pathway is initially stimulated by Wnt receptors on the cell surface. In the activated Wnt pathway, $\beta$-catenin destruction complex is decomposed and the accumulation of $\beta$-catenin in the nucleus is also promoted. Subsequently, these effects can facilitate the expression of Wnt-targeted genes and tumorigenesis $(57,58)$. It was mentioned above that the dysregulation of YAP/TAZ may contribute to human cancer (59). Notably, previous research has discovered an unexpected role of $\mathrm{Wnt} / \beta$-catenin on promoting YAP protein level by activating YAP transcription and interacting with Hippo/YAP. Their interaction considerably contributes to homeostasis, organ repair and tumorigenesis (60). As for colorectal cancer (CRC), $\beta$-catenin was found to associate with TCF/LEF sequence-specific transcriptional factors for the activation of target gene expression. In experiments using human CRC cell lines HCT116, SW620, SW480, RKO, LS174 and HT29, the $\beta$-catenin/TCF4 complex binds to a DNA enhancer element within the first intron of YAP gene to trigger YAP expression (61). It is also noteworthy that as a modulator, the Wnt pathway can stimulate the stabilization of $\beta$-catenin and TAZ (43). Therefore, TAZ activation is speculated as a general feature of the Wnt pathway and is functionally relevant to mediate Wnt biological effects. Furthermore, the stability of TAZ is regulated by the phosphorylation of a
C-terminal phospho-degron via the Hippo pathway and the phosphorylation of an N-terminal degron via GSK3 in the Wnt pathway (62), indicating the substantial connection of the Wnt pathway and Hippo pathway. However, the Wnt pathway has other branches including Wnt/PCP (regulating cell surface polarity), Wnt $/ \mathrm{Ca}^{2+}$ (regulating intracellular calcium signal) and Wnt/ROR1/2. Collectively, these branches are known as the non-canonical Wnt pathway (63). In vertebrates, the non-canonical Wnt pathway can induce osteogenic differentiation and the migration of tumor cells, and inhibit canonical Wnt/ $\beta$-catenin signals (64). Surprisingly, YAP/TAZ are also found to be the downstream effector of the non-canonical Wnt axis, Wnt-FZD/ROR-Ga12/13-Rho GTPases-Lats1/2. In this axis, Wnt5a/b and Wnt3a induce YAP/TAZ activation and nuclear localization, which is independent of canonical Wnt $/ \beta$ catenin signaling. However, upregulation of the expression of YAP/TAZ-TEAD target genes including DKK1, BMP4 and IGFBP4, may lead to Wnt/ $\beta$-catenin inhibition (65).

In terms of biochemical, functional and genetic aspects, YAP/TAZ are integral components of the $\beta$-catenin destruction complex that serves as a cytoplasmic sink for YAP/ TAZ (66). Cytoplasmic YAP may directly sequester $\beta$-catenin into the cytoplasm. Cytoplasmic TAZ may sequester DVL2 to impede its activity in promoting $\beta$-catenin accumulation in response to Wnt stimulation $(67,68)$. In other words, the cytoplasmic YAP/TAZ may have an opposite role here in regulating $\beta$-catenin activity. Hence, the bHippo pathway, known to be involved in tumor suppression, also promotes cytoplasmic localization of YAP/TAZ and inhibits the Wnt pathway. This indicates that the Hippo pathway promotes cytoplasmic retention and degradation of $\beta$-catenin via YAP/TAZ and the Hippo pathway serves as a tumor inhibitor in a novel manner (Fig. 3).

Regulation of YAP/TAZvia GPCR signaling. G protein-coupled receptors (GPCRs) are the largest family of cell surface receptors mediating the responses to a wide range of physiological signals (69). However, abnormal GPCR signaling is involved in tumor development as well. For example, elevated expression of GCPRs such as PAR1 has been demonstrated in high-grade breast cancers, while activated mutations of GPCRs have been found in melanomas and thyroid carcinomas (70-72). As mentioned above, the Hippo pathway is regarded as a tumor suppressor that regulates organ size and tumorigenesis. Based on that, the relationship between activation of YAP/TAZ in cancer and aberrant GPCR signaling has attracted increasing attention (73).

Recently, groundbreaking research has found that serumderived sphingosine-1-phosphate (S1P) and lysophosphatidic acid (LPA) are small-molecule activators of YAP (74). However, $\mathrm{S} 1 \mathrm{P}$ is independent of the core Hippo pathway kinase. It induces YAP nuclear localization through the S1P2 receptor, Rho GTPase activation and F-actin polymerization. Crucially, LPA and S1P activate YAP by binding to their respective GPCRs on the cell surface and by activation of downstream $G$ proteins (75). Namely, the various GPCRs and their agonists importantly serve as Hippo pathway regulators. But notably, activation of GPCRs by epinephrine or glucagon stimulation increases the activity of Lats1/2 kinase, thus resulting in the inhibition of YAP function. In contrast, G12/13- or Gq/11-coupled receptors are activated 


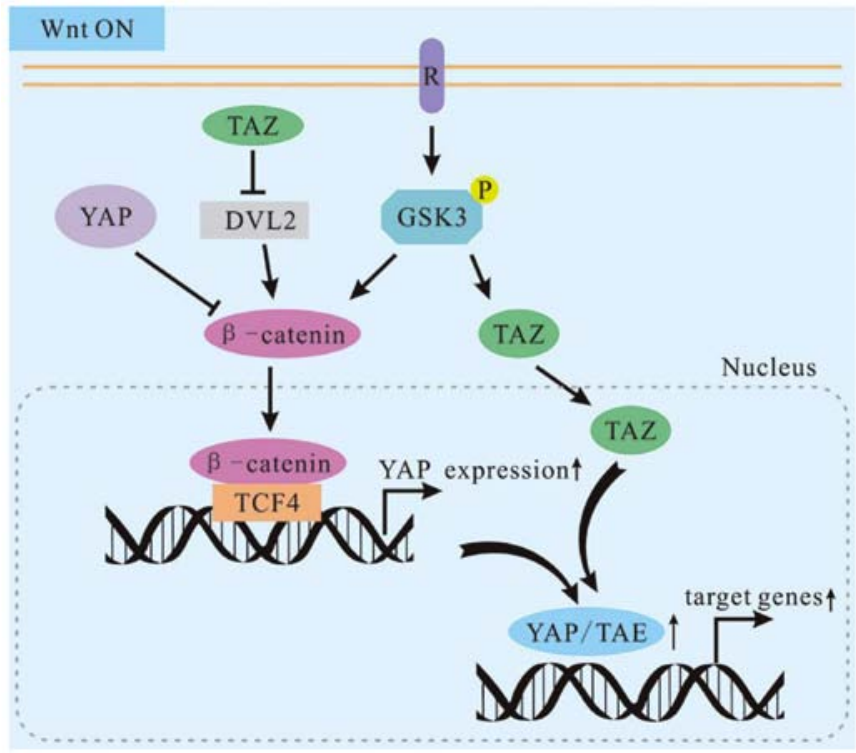

Figure 3. Regulation of the Wnt pathway via YAP/TAZ. The canonical Wnt pathway can decompose $\beta$-catenin destruction complex and promote the accumulation of $\beta$-catenin in the nucleus where the $\beta$-catenin/TCF4 complex binds to a DNA enhancer element within the first intron of the YAP gene to trigger YAP expression in colorectal cancer (CRC) cells. However, in the cytoplasm, YAP/TAZ suppresses the nuclear translocation of $\beta$-catenin, which results in the inhibition of the Wnt pathway. In contrast, the Wnt pathway also stabilizes TAZ through its phosphorylation by GSK3. P, phosphorylation; $\downarrow$, activation; $\perp$, inhibition.

by LPA or S1P, which can inhibit Lats1/2 kinases and result in YAP activation.

However, protein kinase D (PKD) is activated within cells by the stimulation of multiple GPCRs (76). Moreover,
YAP/TAZ are necessary for the stimulation of the proliferative response to GPCR agonists that act via PKD $(77,78)$. It was shown that stimulation of intestinal epithelial IEC-18 cells with angiotensin II (GPCR agonist) induces rapid cytoplasmic accumulation of YAP and concomitant increase of YAP phosphorylation at Ser 127 and Ser 397 (77). However, addiitonal research using human pancreatic cancer cell lines PANC-1 and MiaPaCa-2 revealed that the stimulation of tumor cells with insulin and neurotensin promoted YAP nuclear localization and decreased YAP phosphorylation at Ser 127 and Ser 397, which was mediated by PI3K and PKD. In addition, this would subsequently induce the expression of YAP/TEAD-regulated genes including connective tissue growth factor (CTGF), cysteine-rich angiogenic inducer 61 (CYR61) and CXCL5. siRNA-mediated knockdown of YAP/TAZ, PI3K inhibitor A66 and PKD family inhibitors/siRNAs, would prevent the increase in the mRNA levels of CTGF, CYR61 and CXCL5 in response to insulin and neurotensin stimulation (78). This indicates that PI3K or PKD can promote the crosstalk between insulin receptor and GPCR signaling systems by inducing YAP/TEAD-regulated gene expression in pancreatic cancer cells (Fig. 4).

Nuclear YAP/TAZ in tumorigenesis. The mechanisms of YAP/TAZ translocation are complicated. Apart from the aforementioned pathway, studies on signaling molecules involved in YAP/TAZ nuclear translocation are still underway. For instance, apoptosis-stimulating protein of p53 1 (ASPP1) is able to inhibit the interaction between YAP and Lats1 (79), therefore enhancing nuclear accumulation of YAP/TAZ and YAP/TAZ-dependent transcriptional regulation. Eventually, the nuclear YAP/TAZ could inhibit apoptosis and enhance cell migration.



Figure 4. The effect of GPCR signaling on YAP/TAZ. S1P and LPA inhibit Lats1/2 kinases by activating G12/13- or Gq/11-coupled receptors, resulting in YAP activation and nuclear localization. Conversely, epinephrine and glucagon stimulate GPCRs and increase the activity of Lats1/2 kinase to inactivate YAP (left), Insulin and neurotensin promote striking YAP nuclear localization and decreased YAP phosphorylation by PI3K/PKD in GPCR signaling, which accelerates tumorigenesis. In contrast, the PKD family inhibitors (CRT0066101 and $\mathrm{kb}$ NB 142-70) and PDK siRNA can prohibit this process (medium). Angiotensin II increases the phosphorylation of YAP by stimulating GPCRs, which may induce the cytoplasmic accumulation of YAP and reduce the expression of Ctgf and Areg. GPCRs, G-protein-coupled receptors; S1P, sphingosine-1-phosphate; LPA, lysophosphatidic acid. P, phosphorylation; R, insulin/neurotensin receptor; $\downarrow$, activation; $\perp$, inhibition. 



Figure 5. Nuclear YAP/TAZ promote tumor cell proliferation, inhibit apoptosis and enhance cell migration. (A) S1P and LPA promote YAP/TAZ nuclear translocation by S1P2/Rho GTPase/F-actin polymerization. Moreover, ASPP1 inhibits the interaction between YAP and Lats1, which promotes YAP/TAZ nuclear translocation. As a result, YAP/TAZ can enhance cell proliferation, inhibit apoptosis and increase the activation of mTORC1 by regulating the expression of SLC7A5, DDIT4 and Trail. (B) WIP induces YAP activation and translocation into the nucleus with consequent activation of YAP/TAZ oncogenic targets. In the nucleus, YAP/TAZ both promote the gene expression of NEGR1 and UCA1 that are necessary for maintaining tumorigenic activity. On the contrary, the cell-polarity determinant scribble could disrupt the inhibitory association of TAZ with Mst1/2 and Lats1/2 (left). However, estrogen stimulation activates YAP/TAZ nuclear translocation, and then promotes YAP/TAZ-targeted genes, which enhances cell proliferation, migration, and mitosis (right). S1P, sphingosine-1-phosphate; LPA, lysophosphatidic acid; ASPP1, apoptosis-stimulating protein of p53 1. P, phosphorylation; $\downarrow$, activation; $\perp$, inhibition.

Accumulation of YAP/TAZ broadly participates in the expressionof cancer-related genes.Itwas reported that YAP/TAZ promote cell growth by modulating amino acid signaling to mTORC1 (mammalian target of rapamycin 1) $(80,81)$. In the progress of amino acid-induced mTORC1 activation, particularly under nutrient-limiting conditions, YAP/TAZ potentiate mTORC1 activity by increasing the expression of high-affinity LAT1 (L-type amino acid transporter), a heterodimer of SLC7A5 and SLC3A2. In addition, the YAP/TAZ-TEAD complex directly induces the transcription of SLC7A5, which forms a dimer with SLC3A2 in order to increase LAT1 expression and provide a competitive growth advantage for tumor cells (80). At the same time, YAP/TAZ also inhibit tumor-suppressor genes including DNA-damage-inducible transcript 4 (DDIT4) and TNF-related apoptosis-inducing ligand (TRAIL) that are inhibitors of mTORC1 (81). Herein, we directly address that YAP/TAZ can function as oncogenes by repressing antiproliferative and cell-death genes (Fig. 5A).

In breast cancer, estrogen stimulation activates YAP/TAZ via the $G$ protein-coupled estrogen receptor (GPER) (82). Moreover, GPER mediates the expression of CTGF, CYR61,
EDN1 and EGR1 that are well-established YAP/TAZ target genes (83). In addition, TEAD factors of YAP/TAZ and activator protein-1 (AP-1) form a complex that synergistically activates target genes involved in the control of $\mathrm{S}$ phase entry and mitosis (84). Moreover, wild-type p53 (wtp53) is described as a tumor-suppressor gene, while mutations in this gene (mtp53) occur in many human cancers and promote oncogenic capacity. WIP, phosphorylated by AKT2 in downstream of mtp53, induces YAP/TAZ activation and translocation into the nucleus with consequent activation of YAP/TAZ oncogenic targets (85). From another perspective, TAZ forms a complex with the cell-polarity determinant Scribble. This complex disrupts the inhibitory association of TAZ with the Hippo kinases Mst1/2 and Lats1/2 (86), which promote tumorigenesis. In addition, YAP/TAZ also promote and maintain transforming growth factor- $\beta$ (TGF $\beta$ )-induced tumorigenic phenotypes in late-stage breast cancer cells. The TEAD transcription factors of YAP/TAZ interact with TGF $\beta$ induced SMAD2/3 in the nucleus, eventually promoting the gene expression of NEGR1 and UCA1 that are necessary for maintaining tumorigenic activity (87). Therefore, YAP/TAZ 
are involved in the tumor phenotype and convergence of TAZ/ YAP-TEAD-TGF $\beta$ signals are critical for late-stage breast cancer phenotypes (Fig. 5B).

Nuclear YAP/TAZ also function as transcriptional regulators of the Hippo pathway controlling tumorigenesis and tumor progression in other cancers including lung cancer, oral squamous cell carcinoma (OSCC), and glioblastoma (GBM) $(37,88,89)$. In murine tumor propagating cells (TPCs) marked with Scal and CD24, the knockdown of YAP/TAZ was found to decrease the migration and metastatic potential of lung cancer cells (89). However, the concrete mechanism of YAP/TAZ in TPCs is still under investigation. Moreover, nuclear YAP/TAZ activity drives OSCC cell proliferation, survival and migration. In detail, the transcriptional signature regulated by YAP/TAZ significantly correlates with gene expression changes occurring in human OSCCs including CCNE2, CDK2, CDC6, PCNA, AURKA and PLK4, which has been identified by The Cancer Genome Atlas (TCGA) (37). Additionally, patients with a mesenchymal (MES) gene expression signature exhibit poor overall survival and treatment resistance in glioblastoma. TAZ is directly recruited to a majority of MES gene promoters as a complex with TEAD2, resulting in high-grade tumors with MES features (88). The usual perception is that nuclear YAP/TAZ function as regulators of the Hippo pathway in tumorigenesis; however, when the Hippo pathway is 'on', phosphorylated YAP/TAZ in the cytoplasm act as a retardant for SHP2 nuclear translocator. Namely, only the non-phosphorylated YAP/TAZ promote nuclear translocalization of SHP2. This effect stimulates TCF/LEF- and TEAD-regulated genes via parafibromin (a tumor-suppressor factor) dephosphorylation leading to malignant neoplasms and developmental disorders (90).

\section{YAP/TAZ in oncotherapy}

YAP/TAZ, two transcriptional co-activators of the Hippo pathway, are receiving increased attention owing to their fundamental roles in organ growth and tumor cell proliferation (7). In particular, the participation of YAP/TAZ in cancer indicates the potential of YAP/TAZ as targets for designing anticancer drugs. Fortunately, some inhibitors, directly and indirectly suppressing the activation of YAP/TAZ, have been demonstrated to significantly contribute to effective cancer treatment (91-93). Herein, we highlighted some possible routes for therapeutic intervention.

Regulation of tumor metabolism by targeting YAP/TAZ. The Warburg effect, reprogramming the metabolism of cancer cells towards aerobic glycolysis, supports oncogenic signaling to promote tumor malignancy. Herein, phosphofructokinase 1 (PFK1), regulating the first committed step of glycolysis, binds to the YAP/TAZ transcriptional co-factors TEADs and increases their transcriptional activity (94), whereas 2-deoxyD-glucose (2-DG), as an inhibitor of glycolysis, inhibits YAP/TAZ transcriptional activity and transforming capacity in multiple ways. On the one hand, 2-DG increases the activity of Lats1, the kinase that phosphorylates YAP at Ser 127. On the other hand, 2-DG promotes the interaction between AMP-activated protein kinase (AMPK) and phosphorylated YAP (95). However, tumor cellular energy stress induces
YAP phosphorylation due to the AMPK-dependent activation of Lats, and AMPK can also directly phosphorylate YAP at Ser 94, thus disrupting the YAP-TEAD interaction (96). In other words, energy stress induces YAP cytoplasmic retention and its phosphorylation at Ser 127, ultimately inhibiting YAP transcriptional activity. In addition, AMPK phosphorylates angiomotin-like 1 (AMOTL1) at Ser 793 and increases AMOTL1 steady-state protein levels, which contributes to YAP inhibition (97). Therefore, tumor growth can be suppressed by knockdown of YAP/TAZ or inhibited through the treatment with metformin or AMP mimic aminoimidazole carboxamide ribonucleotide (AICAR) that activates AMPK (95). Metformin, as a widely used oral antidiabetic agent, has been reported to increase AMPK activity and YAP/TAZ phosphorylation in primary mouse hepatocytes after treatment with different doses of metformin for 4 or $8 \mathrm{~h}$. Furthermore, after the treatment of AICAR for $4 \mathrm{~h}$, interaction disruption between YAP and TEAD has also been found in primary mouse hepatocytes with induced YAP/TAZ phosphorylation (96). These findings suggest that aerobic glycolysis endows cancer cells with particular metabolic properties, while energy stress and inhibition of glucose metabolism could inhibit YAP/TAZ. Additionally, these results provide molecular mechanisms for the correlation between cellular metabolism and tumorigenesis in oncotherapy (Fig. 6A).

In addition to aerobic glycolysis mentioned above, the activity of YAP/TAZ was recently found to be regulated by other metabolic pathways such as mevalonate synthesis, nutrient-sensing LKB1-AMPK and TSC-mTOR pathways (98). Among them, the mevalonate-YAP/TAZ axis plays a vital role in proliferation and self-renewal of breast cancer cells, due to the activation of YAP/TAZ promoted by increased levels of mevalonic acid (99). Hence, YAP/TAZ regulation is greatly beyond the regulation by core Hippo kinases, and these new regulatory mechanisms significantly open unexplored routes for therapeutic intervention.

Agents of Hippo, Wnt and GPCR signaling pathways via the regulation of YAP/TAZ. YAP and its paralog protein TAZ are downstream effectors of the Hippo tumor-suppressor pathway and they can be inhibited through phosphorylation-induced cytoplasmic retention and degradation (100). Recently, in polarized Madin-Darby canine kidney (MDCK) cells, some scholars found that knockdown of the AMOT family protein AMOTL2 decreased YAP tight junction localization, reduced the accumulation of nuclear YAP, attenuated YAP phosphorylation, and induced the expression of YAP target gene (101). It was implied that AMOTL2 has an inhibitory effect on YAP. Quickly, the conjecture was confirmed in low metastatic CL1-0 lung cancer cells and in a nude mouse model. AMOT upregulation was transduced from nuclear translocation to cytoplasmic sequestration of YAP/TAZ, leading to decreased expression of Cyr61 (a growth factor) (102). These findings suggest that AMOT is a crucial tumor suppressor and it has bne potential to be a prognostic biomarker and therapeutic target for cancer. Moreover, high mobility group A1 (HMGA1) and its downstream molecule cyclin E2 (CCNE2) exert their stimulatory effect on cell migration by regulating YAP cellular localization and activity, which is called the HMGA1/CCNE2/ YAP axis. However, CDK inhibitors induce the translocation 


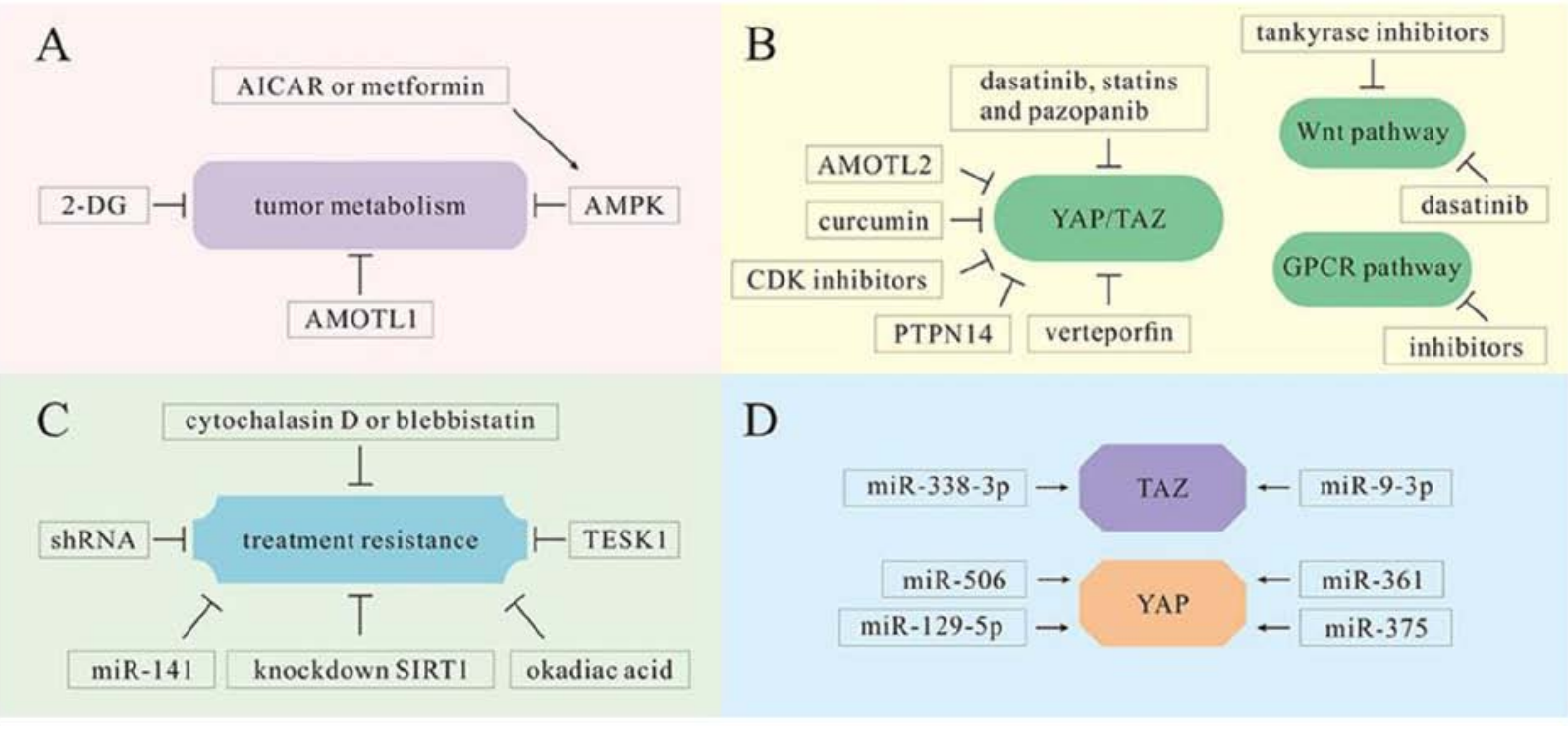

Figure 6. YAP/TAZ as therapeutic targets in multiple mechanisms. (A) Tumor cell metabolism is inhibited by various molecules, including 2-DG, AICAR, metformin and AMOTL1. (B) Various molecules and medicines, including AMOTL2, CDK inhibitors, PTPN14, curcumin, dasatinib, statins, pazopanib and verteporfin, serve as inhibitors of YAP/TAZ in the Hippo pathway and are conducive to cancer treatment. However, tankyrase inhibitors and dasatinib can inhibit the proliferation of tumor cells in the Wnt pathway. There are also inhibitors that can reduce tumor cell proliferation by inhibiting GPCRs. (C) Various molecules and technologies, including cytochalasin D, vlebbistatin, okadiac acid, TESK1, miRNA-141 and shRNAs, can reduce treatment resistance and promote tumor cell sensitivity to oncotherapy. (D) miRNA-338-3p and miR-9-3p can target TAZ in HCC treatment. Other miRNAs, including miR-375, miR-129-5p, miR-506 and miR-361, can target YAP and serve as tumor suppressors. 2-DG, 2-deoxy-D-glucose; AICAR, 5-aminoimidazole-4-carboxamide ribonucleotide; AMOTL1, angiomotin like 1; GPCRs, G-protein-coupled receptors; HCC, hepatocellular carcinoma. $\downarrow$, activation; $\perp$, inhibition.

of YAP from the nucleus to the cytoplasm, which can interdict the axis (103).

The Hippo pathway regulates cellular proliferation and tumorigenesis, which is also controlled by non-receptor tyrosine phosphatase 14 (PTPN14). It was demonstrated that the PPXY domain of PTPN14 binds to WW domain of Kibra, independently and cooperatively promoting the activation and stability of the Lats1 protein (104). Similarly, PPXY motifs of PTPN14 and WW domains of YAP can also form a protein complex, confirming the function of PTPN14 as a negative regulator of YAP. Meanwhile, PTPN14 inhibits YAP-mediated transcriptional activities and sensitizes cancer cells to various anticancer agents such as cisplatin, erlotinib and S12 (105). In addition, in bladder cancer, Kruppel-like factor 5 (KLF5) serves as a modulator to promote cell cycle progression by inducing cyclin D1 expression. Notably, curcumin was found to downregulate the expression of YAP/TAZ and to protect KLF5 protein from proteasome degradation both in human bladder cancer 5637 cells and in xenografted nude mouse models (106). On the basis of these studies, some drug molecules have been verified to play a role in inhibiting YAP in cancer treatment. In uveal melanoma (UM), the activity of cancer-associated $\mathrm{Gq} / 11$ mutants was found to be mediated by YAP. Inversely, the YAP inhibitor verteporfin blocked the growth of UM cells containing Gq/11 mutations (28). Moreover, the results have been also validated by animal experiments. Experiments using breast cancer cell lines (MDA-MB-231, MDA-MB453, HBC-4, HBC-5, MCF-7, BSY-1, ZR-75-1 and SKBR-3) indicates that dasatinib, statins and pazopanib can inhibit the nuclear localization and target gene expression of YAP/TAZ by promoting phosphorylation and proteasomal degradation of YAP/TAZ (107).
As discussed above, cytoplasmic YAP/TAZ, as downstream elements of the $\mathrm{Wnt} / \beta$-catenin cascade, are involved in the degradation and sequestration of $\beta$-catenin in the Wnt pathway (66). YAP/TAZ activation by the Wnt pathway can be prevented by the reactivation of $\beta$-catenin destruction complex with the treatment of tankyrase inhibitors (43). Furthermore, it has been revealed that $\beta$-catenin-active cancers are dependent on a signaling pathway involving YAP1, one of YAP isoforms. The tyrosine kinase YES1 phosphorylates YAP1. Consequently, YAP1 and the transcription factor TBX5 form a complex with $\beta$-catenin. In addition, this complex is essential to the transformation and survival of tumor cells. Moreover, the phosphorylation of YAP1 promotes the localization of this complex and the expression of antiapoptotic genes including BCL2L1 and BIRC5. Importantly, the YES1 inhibitor dasatinib can successfully inhibit the proliferation of $\beta$-catenin-active tumor cells (108).

Furthermore, GPCR is able to upregulate YAP/TAZ activity depending on the specific subset of GPCRs including


also prominent pharmacological targets, suggesting that their selective inhibition may blunt YAP/TAZ activity. However, the 99 mechanisms of targeting YAP/TAZ in these two pathways warrant further research (Fig. 6B).

Agents for treatment resistance through the downregulation of $Y A P / T A Z$. Actin remodeling is the result of oncogenic actin signaling pathway activation or inactivation of several important actin-binding proteins that have tumor-suppressor functions (109). Although the influence of actin remodeling on cancer drug resistance remains unclear, there is research verifying that YAP/TAZ become activated in response to 
Table II. miRNAs as potential inhibitors of YAP/TAZ in oncotherapy.

miRNAs

$\operatorname{miR}-27 / 155$

miRNA-338-3p/9-3p

miR-375/506/361

miR-129-5p
Molecular mechanisms in modulating YAP/TAZ

Silencing of the transcripts of WWC1 (upstream molecules of the Hippo pathway); silencing of the Lats2 gene (inhibitors of YAP/TAZ in the Hippo pathway)

Directly downregulates TAZ

Target YAP

Directly reduces YAP/TAZ expression, inactivates TEAD transcription, downregulates Hippo downstream genes (CTGF and cyclin A) changes in the cytoskeleton. The activation of YAP/TAZ stimulates the actin remodeling of the extracellular matrix (110). BRAF V600E mutant melanoma cells, resistant to the BRAF inhibitor PLX4032 (vemurafenib), exhibit an increase in actin stress fiber formation, which promotes the nuclear accumulation of YAP/TAZ. Actin dynamics regulator TESK1, identified by siRNA library screening, serves as a potential target of PLX4032-resistance in melanoma cells via promoting YAP/TAZ localization (111). Similarly, cytochalasin D or blebbistatin inhibit actomyosin and actin polymerization, respectively, causing YAP/TAZ localization in the cytoplasm and a decrease in the viability of vemurafenib-resistant cells (112). Although post-translational modification, particularly Mst/Lats-mediated phosphorylation at serine 127, has been confirmed to regulate the activity of YAP, its dephosphorylation and acetylation are still unclear. However, researchers have demonstrated that via cisplatin treatment, the catalytic subunit of protein phosphatase-1 (PP1A) interacts with YAP2 and dephosphorylates YAP2, which consequently induces the nuclear accumulation and transcriptional activation of YAP2; whereas, the inhibition of PP1 by okadiac acid (OA) increased the phosphorylation of YAP2 and sensitized ovarian cancer cells to cisplatin treatment both in vivo and in vitro (113). However, YAP2 is deacetylated by Sirtuin 1 (SIRT1), and the deacetylation of YAP2 upregulates YAP2/TEAD binding, leading to YAP2 transcriptional activation and cell growth in hepatocellular carcinoma (HCC) cells; whereas, the knockdown of SIRT1 was found to block the cisplatin-induced nuclear translocation of YAP2 and enhance the chemosensitivity of HCC cells to cisplatin treatment (114). Similarly, it was revealed that miRNA-141 reduced the cisplatin resistance in esophageal squamous cell carcinoma (ESCC) cell lines (KYSE series) via directly targeting the 3'-untranslated region of YAP1 and downregulating YAP1 expression, which has a crucial role in apoptosis induced by DNA-damaging agents (115).

Taxol (paclitaxel) resistance represents a major challenge in breast cancer treatment, but a recent study reports its close relation to TAZ overexpression (85). Short hairpin RNA (shRNA)-mediated knockdown of both Cyr61 and CTGF (downstream transcriptional targets of TAZ) was found to reverse TAZ-induced Taxol resistance in breast cancer cells (85). Likewise, TAZ was found to function as an oncogene in non-small cell lung cancer (NSCLC), and it was knocked down by shRNA in NSCLC cell lines, suppressing tumor cell proliferation and anchorage-independent growth in vitro (116) (Fig. 6C).
MicroRNA (miRNA-associated treatment by modulating YAP/TAZ (Table II). miRNAs, non-coding small RNAs (117-121), are implicated in cell development, cell proliferation, differentiation and apoptosis of cancers (121-128). Recent studies have identified that the hyperexpression of miRNAs are essential for abnormal proliferation and survival of cancer cells though their effect on the Hippo pathway (129). This gives us a hint that miRNAs may provide novel potential for cancer treatment.

In HCC, YAP is involved in HBx-mediated hepatocarcinogenesis. And TAZ is upregulated by preS2. $\mathrm{HBx}$ and preS2, transactivators encoded by $\mathrm{HBV}$, can promote YAP/TAZ at the protein level but not at the mRNA level. Surprisingly, miRNA-338-3p downregulates TAZ and interdicts preS2, while miRNA-338-3p inhibitor restores the expression of TAZ, suggesting that miRNA-338-3p directly targets TAZ (130). In addition, in experiments using HCC cell lines (HepG2, HuH1, HuH7, HLE, HLF, PLC and SKHep1) and HCC clinical samples (frozen HCC tissue), miR-9-3p, as a tumor-suppressor miRNA, was found to target TAZ and reduce TAZ expression. Treatment with miR-9-3p mimic inhibited the cell proliferative ability and downregulated the phosphorylation of Erk1/2, AKT and $\beta$-catenin (cancer promotion factors) (131). Notably, YAP, as a potent oncogenic trigger and independent prognostic risk factor of HCC, was found to be targeted by miR-375. miR-375 was found to be able to inhibit the proliferation and invasion of HCC cells (PLC/PRF/5 and MHCC-97L), and it plays a potential therapeutic role in HCC treatment (132). Coincidentally, miRNAs are also identified to participate in the treatment of ovarian, breast and lung cancer. In experiments using ovarian cancer cell lines (OV56, OVCAR4, TOV-21G, COV362, TOV-112D, SKOV3, COV644, CaOV3, A2780, OV90, COV434 and COV504), miR-129-5p directly repressed YAP and TAZ expression. This repression led to inactivation of TEA domain (TEAD) transcription and the downregulation of Hippo downstream genes including connective tissue growth factor (CTGF) and cyclin A. Thus, miR-129-5p contributes to the subsequent inhibition of cell proliferation, survival and tumorigenicity in ovarian cancer cells (133), but it is not known whether YAP or TAZ is targeted by this miRNA. Recently, the negative correlation of miR-506 with YAP was demonstrated in clinical human breast cancer tissues. A study using MDA-MB231 breast cancer cell line, MCF-7 breast cancer cell line and MCF-10A cell line demonstrated that ectopic miR-506 expression significantly suppressed cell proliferation and affected the cell cycle via targeting YAP (134). Similarly, both in human 
lung cancer cell line A549 and lung cancer tissues, miR-361 was found to be a tumor suppressor in the same way (135). On the other hand, there is increased evidence supporting the function of miRNAs as a target of YAP/TAZ in NSCLC cell lines. In detail, YAP/TAZ inducd transcription of the MCM7 gene and its hosted microRNAs including miR-25, miR-93 and miR106b, thereby promoting cell proliferation in human H1299 and H1975 cells (136). In summary, these studies provide evidence for a novel modulation mechanism of inhibiting the pro-tumorigenic functions of YAP/TAZ through miRNAs. It goes without saying that, miRNAs function as a novel mechanism for YAP/ TAZ regulation. However, their potential in the clinical intervention of human cancers warrants further research (Fig. 6D).

\section{Conclusion}

It is well-known that the Hippo pathway, an evolutionarily conserved signaling pathway, is involved in regulating organ size through controlling the balance of cell proliferation and apoptosis. YAP/TAZ, two key downstream terminal effectors in this pathway, are negatively regulated by its phosphorylation. Accompanied by deactivated Hippo pathway, YAP/TAZ are translocated into the nucleus and promote the transcription of downstream genes through their binding with TEAD. As a result, they facilitate cell proliferation and the amplification of stem cells. Meanwhile, cell apoptosis is also inhibited for the promotion of organ size. However, co-overexpression of YAP/TAZ and deactivation of the Hippo pathway are significantly observed in many malignant cancers, including liver and breast cancer. Moreover, YAP/TAZ also function as modulators of the Wnt pathway and participate in GPCR signaling, playing a vital role in tumorigenesis and tumor progression.

Due to the pivotal effects of YAP/TAZ on organ growth and tumor cell proliferation, it cannot be ignored that targeting YAP/TAZ may be a potential therapeutic strategy for many human cancers. Based on this, direct or indirect inhibition of YAP/TAZ may effectively restrain tumor cell growth, suggesting a novel method for oncotherapy.

\section{Acknowledgements}

Not applicable.

\section{Funding}

The present study was supported by the Sichuan Health and Family Planning Commission Funding (no. 16ZD0253), the Sichuan National Science Research Funding (no. 2015JY0183), funding from the Sichuan Provincial People's Hospital, and a Sichuan Scientific Research Grant for Returned Overseas Chinese Scholars for YW. This study was also funded by the Science and Technology Department of Sichuan Province, China (nos. 16FZ0126, 2011FZ0068 and 14010159) for RT. The study was also supported by the National Key Specialty Construction Project of Clinical Pharmacy (no. 30305030698).

\section{Availability of data and materials}

The datasets used during the present study are available from the corresponding author upon reasonable request.

\section{Authors' contributions}

RT and YW designed the study. SD and HL wrote the manuscript. TL prepared the figures. $\mathrm{HW}$ and $\mathrm{XH}$ reviewed and edited the manuscript. All authors read and approved the manuscript and agree to be accountable for all aspects of the research in ensuring that the accuracy or integrity of any part of the work are appropriately investigated and resolved.

\section{Ethics approval and consent to participate}

This review does not contain any studies with human participants or animals performed by any of the authors.

\section{Consent for publication}

Not applicable.

\section{Competing interests}

The authors declare that they have no conflict of interests.

\section{References}

1. Santucci M, Vignudelli T, Ferrari S, Mor M, Scalvini L, Bolognesi ML, Uliassi E and Costi MP: The Hippo pathway and YAP/TAZ-TEAD protein-protein interaction as targets for regenerative medicine and cancer treatment. J Med Chem 58: 4857-4873, 2015

2. Zhao B, Li L, Lei Q and Guan KL: The Hippo-YAP pathway in organ size control and tumorigenesis: An updated version. Genes Dev 24: 862-874, 2010.

3. Zhao B, Tumaneng K and Guan KL: The Hippo pathway in organ size control, tissue regeneration and stem cell self-renewal. Nat Cell Biol 13: 877-883, 2011.

4. Zhao B, Wei X, Li W, Udan RS, Yang Q, Kim J, Xie J, Ikenoue T, Yu J, Li L, et al: Inactivation of YAP oncoprotein by the Hippo pathway is involved in cell contact inhibition and tissue growth control. Genes Dev 21: 2747-2761, 2007.

5. Lei QY, Zhang H, Zhao B, Zha ZY, Bai F, Pei XH, Zhao S, Xiong Y and Guan KL: TAZ promotes cell proliferation and epithelial-mesenchymal transition and is inhibited by the hippo pathway. Mol Cell Biol 28: 2426-2436, 2008.

6. Cai J, Zhang N, Zheng Y, de Wilde RF, Maitra A and Pan D: The Hippo signaling pathway restricts the oncogenic potential of an intestinal regeneration program. Genes Dev 24: 2383-2388, 2010.

7. Hong $\mathrm{W}$ and Guan KL: The YAP and TAZ transcription co-activators: Key downstream effectors of the mammalian Hippo pathway. Semin Cell Dev Biol 23: 785-793, 2012.

8. Barry ER, Morikawa T, Butler BL, Shrestha K, de la Rosa R, Yan KS, Fuchs CS, Magness ST, Smits R, Ogino S, et al: Restriction of intestinal stem cell expansion and the regenerative response by YAP. Nature 493: 106-110, 2013.

9. Ma Y, Yang Y, Wang F, Wei Q and Qin H: Hippo-YAP signaling pathway: A new paradigm for cancer therapy. Int J Cancer 137: 2275-2286, 2015.

10. Mo JS, Park HW and Guan KL: The Hippo signaling pathway in stem cell biology and cancer. EMBO Rep 15: 642-656, 2014.

11. Pan D: The hippo signaling pathway in development and cancer. Dev Cell 19: 491-505, 2010

12. Wang L, Shi S, Guo Z, Zhang X, Han S, Yang A, Wen W and Zhu Q: Overexpression of YAP and TAZ is an independent predictor of prognosis in colorectal cancer and related to the proliferation and metastasis of colon cancer cells. PLoS One 8: e65539, 2013.

13. Halder G and Johnson RL: Hippo signaling: Growth control and beyond. Development 138: 9-22, 2011.

14. Moroishi T, Hansen CG and Guan KL: The emerging roles of YAP and TAZ in cancer. Nat Rev Cancer 15: 73-79, 2015.

15. Piccolo S, Dupont S and Cordenonsi M: The biology of YAP/ TAZ: Hippo signaling and beyond. Physiol Rev 94: 1287-1312, 2014. 
16. Moroishi T, Park HW, Qin B, Chen Q, Meng Z, Plouffe SW, Taniguchi K, Yu FX, Karin M,Pan D, et al: A YAP/TAZ-induced feedback mechanism regulates Hippo pathway homeostasis. Genes Dev 29: 1271-1284, 2015.

17. Wang L, Chen Z, Wang Y, Chang D, Su L, Guo Y and Liu C: TR1 promotes cell proliferation and inhibits apoptosis through cyclin A and CTGF regulation in non-small cell lung cancer. Tumour Biol 35: 463-468, 2014.

18. Nishioka N, Inoue K, Adachi K, Kiyonari H, Ota M, Ralston A, Yabuta N, Hirahara S, Stephenson RO, Ogonuki N, et al: The Hippo signaling pathway components Lats and Yap pattern Tead4 activity to distinguish mouse trophectoderm from inner cell mass. Dev Cell 16: 398-410, 2009.

19. Xin M, Kim Y, Sutherland LB, Murakami M, Qi X, McAnally J, Porrello ER, Mahmoud AI, Tan W, Shelton JM, et al: Hippo pathway effector Yap promotes cardiac regeneration. Proc Natl Acad Sci USA 110: 13839-13844, 2013.

20. Zaidi SK, Sullivan AJ, Medina R, Ito Y, van Wijnen AJ, Stein JL, Lian JB and Stein GS: Tyrosine phosphorylation controls Runx2mediated subnuclear targeting of YAP to repress transcription. EMBO J 23: 790-799, 2004.

21. Zhou Z, Hu T, Xu Z, Lin Z, Zhang Z, Feng T, Zhu L, Rong Y, Shen H, Luk JM, et al: Targeting Hippo pathway by specific interruption of YAP-TEAD interaction using cyclic YAP-like peptides. FASEB J 29: 724-732, 2015.

22. Varelas X: The Hippo pathway effectors TAZ and YAP in development, homeostasis and disease. Development 141: 1614-1626, 2014.

23. Han SX, Bai E, Jin GH, He CC, Guo XJ, Wang LJ, Li M, Ying X and Zhu Q: Expression and clinical significance of YAP, TAZ, and AREG in hepatocellular carcinoma. J Immunol Res 2014 261365, 2014.

24. Sudol M, Bork P, Einbond A, Kastury K, Druck T, Negrini M, Huebner K and Lehman D: Characterization of the mammalian YAP (Yes-associated protein) gene and its role in defining a novel protein module, the WW domain. J Biol Chem 270: 14733-14741, 1995.

25. Morin-Kensicki EM, Boone BN, Howell M, Stonebraker JR, Teed J, Alb JG, Magnuson TR, O'Neal W and Milgram SL: Defects in yolk sac vasculogenesis, chorioallantoic fusion, and embryonic axis elongation in mice with targeted disruption of Yap65. Mol Cell Biol 26: 77-87, 2006.

26. Muramatsu T, Imoto I, Matsui T, Kozaki K, Haruki S, Sudol M, Shimada Y, Tsuda H, Kawano T and Inazawa J: YAP is a candidate oncogene for esophageal squamous cell carcinoma. Carcinogenesis 32: 389-398, 2011.

27. Gaffney CJ, Oka T, Mazack V, Hilman D, Gat U, Muramatsu T, Inazawa J, Golden A, Carey DJ, Farooq A, et al: Identification, basic characterization and evolutionary analysis of differentially spliced mRNA isoforms of human YAP1 gene. Gene 509: 215-222, 2012.

28. Yu FX, Luo J, Mo JS, Liu G, Kim YC, Meng Z, Zhao L, Peyman G, Ouyang H, Jiang W, et al: Mutant Gq/11 promote uveal melanoma tumorigenesis by activating YAP. Cancer Cell 25: 822-830, 2014

29. Hall CA, Wang R, Miao J, Oliva E, Shen X, Wheeler T, Hilsenbeck SG, Orsulic S and Goode S: Hippo pathway effector Yap is an ovarian cancer oncogene. Cancer Res 70: 8517-8525, 2010.

30. Sudol M: Yes-associated protein (YAP65) is a proline-rich phosphoprotein that binds to the $\mathrm{SH} 3$ domain of the Yes protooncogene product. Oncogene 9: 2145-2152, 1994.

31. Kanai F, Marignani PA, Sarbassova D, Yagi R, Hall RA, Donowitz M, Hisaminato A, Fujiwara T, Ito Y, Cantley LC, et al: TAZ: A novel transcriptional co-activator regulated by interactions with 14-3-3 and PDZ domain proteins. EMBO J 19: 6778-6791, 2000.

32. Li PD, Wang XJ, Shan Q, Wu YH and Wang Z: Evaluation of TAZ expression and its effect on tumor invasion and metastasis in human glioma. Asian Pac J Trop Med 7: 757-760, 2014.

33. Nagaraj R, Gururaja-Rao S, Jones KT, Slattery M, Negre N, Braas D, Christofk H, White KP, Mann R and Banerjee U: Control of mitochondrial structure and function by the Yorkie/ YAP oncogenic pathway. Genes Dev 26: 2027-2037, 2012.

34. Guo L and Teng L: YAP/TAZ for cancer therapy: Opportunities and challenges (Review). Int J Oncol 46: 1444-1452, 2015.

35. Oka T and Sudol M: Nuclear localization and pro-apoptotic signaling of YAP2 require intact PDZ-binding motif. Genes Cells 14: 607-615, 2009.
36. Sawada A, Kiyonari H, Ukita K, Nishioka N, Imuta Y and Sasaki H: Redundant roles of Tead1 and Tead 2 in notochord development and the regulation of cell proliferation and survival. Mol Cell Biol 28: 3177-3189, 2008.

37. Hiemer SE, Zhang L, Kartha VK, Packer TS, Almershed M, Noonan V, Kukuruzinska M, Bais MV, Monti S and Varelas X: A YAP/TAZ-Regulated Molecular Signature Is Associated with Oral Squamous Cell Carcinoma. Mol Cancer Res 13: 957-968, 2015.

38. Chan SW, Lim CJ, Chong YF, Pobbati AV, Huang C and Hong W: Hippo pathway-independent restriction of TAZ and YAP by angiomotin. J Biol Chem 286: 7018-7026, 2011.

39. Liu C, Huang W and Lei Q: Regulation and function of the TAZ transcription co-activator. Int J Biochem Mol Biol 2: 247-256, 2011.

40. Yue G, Sun X, Gimenez-Capitan A, Shen J, Yu L, Teixido C, Guan W, Rosell R, Liu B and Wei J: TAZ is highly expressed in gastric signet ring cell carcinoma. BioMed Res Int 2014: 393064, 2014.

41. Aqeilan RI, Donati V, Palamarchuk A, Trapasso F, Kaou M, Pekarsky Y, Sudol M and Croce CM: WW domain-containing proteins, WWOX and YAP, compete for interaction with ErbB-4 and modulate its transcriptional function. Cancer Res 65: 6764-6772, 2005.

42. Donzelli S, Strano S and Blandino G: YAP and p73: A matter of mutual specificity in tumor suppression. In: The Hippo Signaling Pathway and Cancer. Springer, New York, pp147-172, 2013.

43. Azzolin L, Zanconato F, Bresolin S, Forcato M, Basso G, Bicciato S, Cordenonsi M and Piccolo S: Role of TAZ as mediator of Wnt signaling. Cell 151: 1443-1456, 2012.

44. Low BC, Pan CQ, Shivashankar GV, Bershadsky A, Sudol M and Sheetz M: YAP/TAZ as mechanosensors and mechanotransducers in regulating organ size and tumor growth. FEBS Lett 588: 2663-2670, 2014.

45. Acerbi I, Cassereau L, Dean I, Shi Q, Au A, Park C, Chen YY, Liphardt J, Hwang ES and Weaver VM: Human breast cancer invasion and aggression correlates with ECM stiffening and immune cell infiltration. Integr Biol 7: 1120-1134, 2015.

46. Calvo F, Ege N, Grande-Garcia A, Hooper S, Jenkins RP, Chaudhry SI, Harrington K, Williamson P, Moeendarbary E, Charras G, et al: Mechanotransduction and YAP-dependent matrix remodelling is required for the generation and maintenance of cancer-associated fibroblasts. Nat Cell Biol 15: 637-646, 2013.

47. Halder G, Dupont S and Piccolo S: Transduction of mechanical and cytoskeletal cues by YAP and TAZ. Nat Rev Mol Cell Biol 13: 591-600, 2012.

48. Aragona M, Panciera T, Manfrin A, Giulitti S, Michielin F, Elvassore N, Dupont S and Piccolo S: A mechanical checkpoint controls multicellular growth through YAP/TAZ regulation by actin-processing factors. Cell 154: 1047-1059, 2013.

49. Gibson WT and Gibson MC: Cell topology, geometry, and morphogenesis in proliferating epithelia. Curr Top Dev Biol 89: 87-114, 2009

50. Vogel V and Sheetz M: Local force and geometry sensing regulate cell functions. Nat Rev Mol Cell Biol 7: 265-275, 2006.

51. Furukawa KT, Yamashita K, Sakurai N and Ohno S: The epithelial circumferential actin belt regulates YAP/TAZ through nucleocytoplasmic shuttling of merlin. Cell Reports 20: 1435-1447, 2017.

52. Matsui Y and Lai ZC: Mutual regulation between Hippo signaling and actin cytoskeleton. Protein Cell 4: 904-910, 2013.

53. Zhao B, Li L, Wang L, Wang CY, Yu J and Guan KL: Cell detachment activates the Hippo pathway via cytoskeleton reorganization to induce anoikis. Genes Dev 26: 54-68, 2012.

54. Wada K, Itoga K, Okano T, Yonemura S and Sasaki H: Hippo pathway regulation by cell morphology and stress fibers. Development 138: 3907-3914, 2011.

55. Sansores-Garcia L, Bossuyt W, Wada K, Yonemura S, Tao C, Sasaki $\mathrm{H}$ and Halder G: Modulating F-actin organization induces organ growth by affecting the Hippo pathway. EMBO J 30: 2325-2335, 2011 .

56. Talele NP, Fradette J, Davies JE, Kapus A and Hinz B: Expression of $\alpha$-smooth muscle actin determines the fate of mesenchymal stromal cells. Stem Cell Reports 4: 1016-1030, 2015.

57. Miyabara S, Yuda Y, Kasashima Y, Kuwano A and Arai K: Regulation of Tenomodulin Expression Via Wnt/ $\beta$-catenin Signaling in Equine Bone Marrow-derived Mesenchymal Stem Cells. J Equine Sci 25: 7-13, 2014.

58. Lustig B and Behrens J: The Wnt signaling pathway and its role in tumor development. J Cancer Res Clin Oncol 129: 199-221, 2003. 
59. Yu FX, Zhao B and Guan KL: Hippo pathway in organ size control, tissue homeostasis, and cancer. Cell 163: 811-828, 2015.

60. Konsavage WM Jr and Yochum GS: Intersection of Hippo/YAP and Wnt/ $\beta$-catenin signaling pathways. Acta Biochim Biophys Sin (Shanghai) 45: 71-79, 2013

61. Konsavage WM Jr, Kyler SL, Rennoll SA, Jin G and Yochum GS: $\mathrm{Wnt} / \beta$-catenin signaling regulates Yes-associated protein (YAP) gene expression in colorectal carcinoma cells. J Biol Chem 287: 11730-11739, 2012.

62. Huang W, Lv X, Liu C, Zha Z, Zhang H, Jiang Y, Xiong Y, Lei QY and Guan KL: The N-terminal phosphodegron targets TAZ/WWTR1 protein for SCF $\beta$-TrCP-dependent degradation in response to phosphatidylinositol 3-kinase inhibition. J Biol Chem 287: 26245-26253, 2012.

63. Nishita M, Endo M and Minami Y: Regulation of cellular responses by non-canonical Wnt signaling. Clin Calcium 23: 809-815, 2013 (In Japanese)

64. Korswagen HC: Canonical and non-canonical Wnt signaling pathways in Caenorhabditis elegans: Variations on a common signaling theme. BioEssays 24: 801-810, 2002.

65. Park HW, Kim YC, Yu B, Moroishi T, Mo JS, Plouffe SW, Meng Z, Lin KC, Yu FX, Alexander CM, et al: Alternative Wnt Signaling Activates YAP/TAZ. Cell 162: 780-794, 2015

66. Azzolin L, Panciera T, Soligo S, Enzo E, Bicciato S, Dupont S, Bresolin S, Frasson C, Basso G, Guzzardo V, et al: YAP/TAZ incorporation in the $\beta$-catenin destruction complex orchestrates the Wnt response. Cell 158: 157-170, 2014.

67. Varelas X, Miller BW, Sopko R, Song S, Gregorieff A, Fellouse FA, Sakuma R, Pawson T, Hunziker W, McNeill H, et al: The Hippo pathway regulates Wnt/beta-catenin signaling. Dev Cell 18: 579-591, 2010.

68. Imajo M, Miyatake K, Iimura A, Miyamoto A and Nishida E: A molecular mechanism that links Hippo signalling to the inhibition of Wnt/ $\beta$-catenin signalling. EMBO J 31: 1109-1122, 2012.

69. Lappano R and Maggiolini M: G protein-coupled receptors: Novel targets for drug discovery in cancer. Nat Rev Drug Discov 10: 47-60, 2011.

70. Prickett TD, Wei X, Cardenas-Navia I, Teer JK, Lin JC, Walia V, Gartner J, Jiang J, Cherukuri PF, Molinolo A, et al: Exon capture analysis of $\mathrm{G}$ protein-coupled receptors identifies activating mutations in GRM3 in melanoma. Nat Genet 43: 1119-1126, 2011

71. Paschke R and Ludgate $\mathrm{M}$ : The thyrotropin receptor in thyroid diseases. N Engl J Med 337: 1675-1681, 1997.

72. Hernández NA, Correa E, Avila EP, Vela TA and Pérez VM: PAR1 is selectively over expressed in high grade breast cancer patients: A cohort study. J Transl Med 7: 47, 2009.

73. Guo $X$ and Zhao B: Integration of mechanical and chemical signals by YAP and TAZ transcription coactivators. Cell Biosci 3: 33,2013

74. Miller E, Yang J, DeRan M, Wu C, Su AI, Bonamy GMC, Liu J, Peters EC and $\mathrm{Wu} \mathrm{X}$ : Identification of serum-derived sphingosine-1-phosphate as a small molecule regulator of YAP. Chem Biol 19: 955-962, 2012.

75. Yu FX, Zhao B, Panupinthu N, Jewell JL, Lian I, Wang LH, Zhao J, Yuan H, Tumaneng K, Li H, et al: Regulation of the Hippo-YAP pathway by G-protein-coupled receptor signaling. Cell 150: 780-791, 2012.

76. Waldron RT, Innamorati G, Torres-Marquez ME, Sinnett-Smith J and Rozengurt E; RT W: Differential PKC-dependent and -independent PKD activation by $\mathrm{G}$ protein $\alpha$ subunits of the $\mathrm{Gq}$ family: Selective stimulation of PKD $\operatorname{Ser}^{748}$ autophosphorylation by Gaq. Cell Signal 24: 914-921, 2012.

77. Wang J, Sinnett-Smith J, Stevens JV, Young SH and Rozengurt E: Biphasic regulation of Yes-associated protein (YAP) cellular localization, phosphorylation, and activity by $G$ protein-coupled receptor agonists in intestinal epithelial cells: A novel role for protein kinase D (PKD). J Biol Chem 291: 17988-18005, 2016.

78. Hao F, Xu Q, Zhao Y, Stevens JV, Young SH, Sinnett-Smith J and Rozengurt E: Insulin receptor and GPCR crosstalk stimulates YAP via PI3K and PKD in pancreatic cancer cells. Mol Cancer Res 15: 929-941, 2017.

79. Vigneron AM, Ludwig RL and Vousden KH: Cytoplasmic ASPP1 inhibits apoptosis through the control of YAP. Genes Dev 24: 2430-2439, 2010.

80. Hansen CG, Ng YL, Lam WL, Plouffe SW and Guan KL: The Hippo pathway effectors YAP and TAZ promote cell growth by modulating amino acid signaling to mTORC1. Cell Res 25: 1299-1313, 2015.

81. Kim M, Kim T, Johnson RL and Lim DS: Transcriptional co-repressor function of the hippo pathway transducers YAP and TAZ. Cell Reports 11: 270-282, 2015.
82. Zhou X, Wang S, Wang Z, Feng X, Liu P, Lv XB, Li F, Yu FX, Sun Y, Yuan H, et al: Estrogen regulates Hippo signaling via GPER in breast cancer. J Clin Invest 125: 2123-2135, 2015.

83. Zhao B, Ye X, Yu J, Li L, Li W, Li S, Yu J, Lin JD, Wang CY, Chinnaiyan AM, et al: TEAD mediates YAP-dependent gene induction and growth control. Genes Dev 22: 1962-1971, 2008.

84. Zanconato F, Forcato M, Battilana G, Azzolin L, Quaranta E, Bodega B, Rosato A, Bicciato S, Cordenonsi M and Piccolo S: Genome-wide association between YAP/TAZ/TEAD and AP-1 at enhancers drives oncogenic growth. Nat Cell Biol 17: 1218-1227, 2015

85. Escoll M, Gargini R, Cuadrado A, Anton IM and Wandosell F Mutant p53 oncogenic functions in cancer stem cells are regulated by WIP through YAP/TAZ. Oncogene 36: 3515-3527, 2017.

86. Cordenonsi M, Zanconato F, Azzolin L, Forcato M, Rosato A, Frasson C, Inui M, Montagner M, Parenti AR, Poletti A, et al: The Hippo transducer TAZ confers cancer stem cell-related traits on breast cancer cells. Cell 147: 759-772, 2011.

87. Hiemer SE, Szymaniak AD and Varelas X: The transcriptional regulators TAZ and YAP direct transforming growth factor $\beta$-induced tumorigenic phenotypes in breast cancer cells. J Biol Chem 289: 13461-13474, 2014

88. Bhat KP, Salazar KL, Balasubramaniyan V, Wani K, Heathcock L, Hollingsworth F, James JD, Gumin J, Diefes KL, Kim SH, et al: The transcriptional coactivator TAZ regulates mesenchymal differentiation in malignant glioma. Genes Dev 25: 2594-2609, 2011.

89. Lau AN, Curtis SJ, Fillmore CM, Rowbotham SP, Mohseni M, Wagner DE, Beede AM, Montoro DT, Sinkevicius KW, Walton ZE, et al: Tumor-propagating cells and Yap/Taz activity contribute to lung tumor progression and metastasis. EMBO J 33: 468-481, 2014

90. Tsutsumi R, Masoudi M, Takahashi A, Fujii Y, Hayashi T, Kikuchi I, Satou Y, Taira M and Hatakeyama M: YAP and TAZ, Hippo signaling targets, act as a rheostat for nuclear SHP2 function. Dev Cell 26: 658-665, 2013

91. Bae JS, Kim SM and Lee H: The Hippo signaling pathway provides novel anti-cancer drug targets. Oncotarget 8 16084-16098, 2017.

92. Tao Y, Cai F, Shan L, Jiang H, Ma L and Yu Y: The Hippo signaling pathway: An emerging anti-cancer drug target. Discov Med 24: 7-18, 2017.

93. Zanconato F, Battilana G, Cordenonsi $\mathrm{M}$ and Piccolo $\mathrm{S}$ YAP/TAZ as therapeutic targets in cancer. Curr Opin Pharmacol 29: $26-33,2016$

94. Enzo E, Santinon G, Pocaterra A, Aragona M, Bresolin S, Forcato M, Grifoni D, Pession A, Zanconato F, Guzzo G, et al: Aerobic glycolysis tunes YAP/TAZ transcriptional activity. EMBO J 34: 1349-1370, 2015

95. Wong W: Keeping cells from getting Hippo-sized under energy stress. Sci Signal 8: ec108, 2015

96. Mo JS, Meng Z, Kim YC, Park HW, Hansen CG, Kim S, Lim DS and Guan KL: Cellular energy stress induces AMPK-mediated regulation of YAP and the Hippo pathway. Nat Cell Biol 17: 500-510, 2015.

97. DeRan M, Yang J, Shen CH, Peters EC, Fitamant J, Chan P, Hsieh M,Zhu S, Asara JM,Zheng B, et al: Energy stress regulates hippo-YAP signaling involving AMPK-mediated regulation of angiomotin-like 1 protein. Cell Reports 9: 495-503, 2014

98. Santinon G, Pocaterra A and Dupont S: Control of YAP/TAZ activity by metabolic and nutrient-sensing pathways. Trends Cell Biol 26: 289-299, 2016.

99. Sorrentino G, Ruggeri N, Specchia V, Cordenonsi M, Mano M, Dupont S, Manfrin A, Ingallina E, Sommaggio R, Piazza S, et al: Metabolic control of YAP and TAZ by the mevalonate pathway. Nat Cell Biol 16: 357-366, 2014.

100. Nallet-Staub F, Marsaud V, Li L, Gilbert C, Dodier S, Bataille V, Sudol M, Herlyn M and Mauviel A: Pro-invasive activity of the Hippo pathway effectors YAP and TAZ in cutaneous melanoma. J Invest Dermatol 134: 123-132, 2014

101. Zhao B, Li L, Lu Q, Wang LH, Liu CY, Lei Q and Guan KL: Angiomotin is a novel Hippo pathway component that inhibits YAP oncoprotein. Genes Dev 25: 51-63, 2011.

102. Hsu YL, Hung JY, Chou SH, Huang MS, Tsai MJ, Lin YS, Chiang SY, Ho YW, Wu CY and Kuo PL: Angiomotin decreases lung cancer progression by sequestering oncogenic YAP/TAZ and decreasing Cyr61 expression. Oncogene 34: 4056-4068, 2015. 
103. Pegoraro S, Ros G, Ciani Y, Sgarra R, Piazza S and Manfioletti G: A novel HMGA1-CCNE2-YAP axis regulates breast cancer aggressiveness. Oncotarget 6: 19087-19101, 2015.

104. Wilson KE, Li YW, Yang N, Shen H, Orillion AR and Zhang J: PTPN14 forms a complex with Kibra and LATS1 proteins and negatively regulates the YAP oncogenic function. J Biol Chem 289: 23693-23700, 2014

105. Huang JM, Nagatomo I, Suzuki E, Mizuno T, Kumagai T, Berezov A, Zhang H, Karlan B, Greene MI and Wang Q: YAP modifies cancer cell sensitivity to EGFR and survivin inhibitors and is negatively regulated by the non-receptor type protein tyrosine phosphatase 14. Oncogene 32: 2220-2229, 2013.

106. Gao Y, Shi Q, Xu S, Du C, Liang L, Wu K, Wang K, Wang X, Chang LS, He D, et al: Curcumin promotes KLF5 proteasome degradation through downregulating YAP/TAZ in bladder cancer cells. Int J Mol Sci 15: 15173-15187, 2014.

107. Oku Y, Nishiya N, Shito T, Yamamoto R, Yamamoto Y, Oyama $\mathrm{C}$ and Uehara $\mathrm{Y}$ : Small molecules inhibiting the nuclear localization of YAP/TAZ for chemotherapeutics and chemosensitizers against breast cancers. FEBS Open Bio 5: 542-549, 2015

108. Rosenbluh J, Nijhawan D, Cox AG, Li X, Neal JT, Schafer EJ, Zack TI, Wang X, Tsherniak A, Schinzel AC, et al: $\beta$-Catenindriven cancers require a YAP1 transcriptional complex for survival and tumorigenesis. Cell 151: 1457-1473, 2012.

109. Rao J and Li N: Microfilament actin remodeling as a potential target for cancer drug development. Curr Cancer Drug Targets 4: 345-354, 2004

110. Morikawa Y, Zhang M, Heallen T, Leach J, Tao G, Xiao Y, Bai Y, Li W, Willerson JT and Martin JF: Actin cytoskeletal remodeling with protrusion formation is essential for heart regeneration in Hippo-deficient mice. Sci Signal 8: ra41, 2015.

111. Kim MH, Kim J, Hong H, Lee SH, Lee JK, Jung E and Kim J: Actin remodeling confers BRAF inhibitor resistance to melanoma cells through YAP/TAZ activation. EMBO J 35 462-478, 2016.

112. Ferrarelli LK: Actin against BRAF inhibitors. Sci Signal 9: ec51,2016.

113. Wang P, Bai Y, Song B, Wang Y, Liu D, Lai Y, Bi X and Yuan Z: PP1A-mediated dephosphorylation positively regulates YAP2 activity. PLoS One 6: e24288, 2011

114. Mao B, Hu F, Cheng J, Wang P, Xu M, Yuan F, Meng S, Wang Y, Yuan Z and Bi W: SIRT1 regulates YAP2-mediated cell proliferation and chemoresistance in hepatocellular carcinoma. Oncogene 33: 1468-1474, 2014.

115. Imanaka Y, Tsuchiya S, Sato F, Shimada Y, Shimizu K and Tsujimoto G: MicroRNA-141 confers resistance to cisplatininduced apoptosis by targeting YAP1 in human esophageal squamous cell carcinoma. J Hum Genet 56: 270-276, 2011

116.Zhou Z, Hao Y, Liu N, Raptis L, Tsao MS and Yang X: TAZ is a novel oncogene in non-small cell lung cancer. Oncogene 30: 2181-2186, 2011.

117. Anastasiadou E, Jacob LS and Slack FJ: Non-coding RNA networks in cancer. Nat Rev Cancer 18: 5-18, 2017.

118. Esteller M: Non-coding RNAs in human disease. Nat Rev Genet 12: 861-874, 2011

119. He L and Hannon GJ: MicroRNAs: Small RNAs with a big role in gene regulation. Nat Rev Genet 5: 522-531, 2004.

120. Lu J, Getz G, Miska EA, Alvarez-Saavedra E, Lamb J, Peck D, Sweet-Cordero A, Ebert BL, Mak RH, Ferrando AA, et al: MicroRNA expression profiles classify human cancers. Nature 435: 834-838, 2005 .

121. Stefani G and Slack FJ: Small non-coding RNAs in animal development. Nat Rev Mol Cell Biol 9: 219-230, 2008.

122. Sassen S, Miska EA and Caldas C: MicroRNA: Implications for cancer. Virchows Arch 452: 1-10, 2008.
123. Kim HS, Lee KS, Bae HJ, Eun JW, Shen Q, Park SJ, Shin WC, Yang HD, Park M, Park WS, et al: MicroRNA-31 functions as a tumor suppressor by regulating cell cycle and epithelialmesenchymal transition regulatory proteins in liver cancer. Oncotarget 6: 8089-8102, 2015.

124. Lin S and Gregory RI: MicroRNA biogenesis pathways in cancer. Nat Rev Cancer 15: 321-333, 2015.

125. Nishikawa R, Goto Y, Kojima S, Enokida H, Chiyomaru T, Kinoshita T, Sakamoto S, Fuse M, Nakagawa M, Naya Y, et al: Tumor-suppressive microRNA-29s inhibit cancer cell migration and invasion via targeting LAMC1 in prostate cancer. Int $\mathrm{J}$ Oncol 45: 401-410, 2014.

126. Nishikawa R, Goto Y, Sakamoto S, Chiyomaru T, Enokida H, Kojima S, Kinoshita T, Yamamoto N, Nakagawa M, Naya Y, et al: Tumor-suppressive microRNA-218 inhibits cancer cell migration and invasion via targeting of LASP1 in prostate cancer. Cancer Sci 105: 802-811, 2014.

127. Ouyang H, Gore J, Deitz S and Korc M: microRNA-10b enhances pancreatic cancer cell invasion by suppressing TIP30 expression and promoting EGF and TGF- $\beta$ actions. Oncogene 33: 4664-4674, 2014

128. Cirilo PDR, de Sousa Andrade LN, Corrêa BRS, Qiao M, Furuya TK, Chammas R and Penalva LOF: MicroRNA-195 acts as an anti-proliferative miRNA in human melanoma cells by targeting Prohibitin 1. BMC Cancer 17: 750, 2017.

129. Halytskiy V: Shifts in miRNA expression pattern can lead to the loss of contact inhibition in breast cancer cells. Eur J Cancer 57 (Suppl 2): S116 2016.

130. Liu P, Zhang H, Liang X, Ma H, Luan F, Wang B, Bai F, Gao L and Ma C: HBV preS2 promotes the expression of TAZ via miRNA-338-3p to enhance the tumorigenesis of hepatocellular carcinoma. Oncotarget 6: 29048-29059, 2015.

131. Higashi T, Hayashi H, Ishimoto T, Takeyama H, Kaida T, Arima K, Taki K, Sakamoto K, Kuroki H, Okabe H, et al: miR-9-3p plays a tumour-suppressor role by targeting TAZ (WWTR1) in hepatocellular carcinoma cells. Br J Cancer 113: 252-258, 2015

132. Liu AM, Poon RT and Luk JM: MicroRNA-375 targets Hipposignaling effector YAP in liver cancer and inhibits tumor properties. Biochem Biophys Res Commun 394: 623-627, 2010.

133. Tan G, Cao X, Dai Q, Zhang B, Huang J, Xiong S, Zhang Y, Chen W, Yang J and Li H: A novel role for microRNA-129-5p in inhibiting ovarian cancer cell proliferation and survival via direct suppression of transcriptional co-activators YAP and TAZ. Oncotarget 6: 8676-8686, 2015.

134. Hua K, Yang W, Song H, Song J, Wei C, Li D and Fang L: Up-regulation of miR-506 inhibits cell growth and disrupt the cell cycle by targeting YAP in breast cancer cells. Int J Clin Exp Med 8: 12018-12027, 2015.

135.Zhang S, Liu Z, Wu L and Wang Y: MiR-361 targets Yes-associated protein (YAP) mRNA to suppress cell proliferation in lung cancer. Biochem Biophys Res Commun 492: 468-473, 2017.

136. Lo Sardo F, Forcato M, Sacconi A, Capaci V, Zanconato F, Di Agostino S, Del Sal G, Pandolfi PP, Strano S, Bicciato S, et al: MCM7 and its hosted miR-25, 93 and 106b cluster elicit YAP/TAZ oncogenic activity in lung cancer. Carcinogenesis 38: $64-75,2017$.

This work is licensed under a Creative Commons Attribution-NonCommercial-NoDerivatives 4.0 International (CC BY-NC-ND 4.0) License. 